\title{
Assessing the market potential of electric bicycles and ICT for low carbon school travel: a case study in the Smart City of ÁGUEDA
}

\author{
Elisabete Arsenio ${ }^{1} \cdot$ Joana V. Dias $^{2} \cdot$ Sofia Azeredo Lopes ${ }^{3} \cdot$ Helena Iglésias Pereira ${ }^{4}$ \\ Received: 6 January 2017 / Accepted: 4 December 2017 / Published online: 20 January 2018 \\ (C) The Author(s) 2018. This article is an open access publication
}

\begin{abstract}
Introduction Electric bikes (e-bikes) may help in transport decarbonisation in European cities. To fully assess the market potential of e-bikes, further research is needed to understand users' preferences and the range of factors that can contribute to people to shift from car use to low carbon vehicles such as e-bikes.

This paper is built on the Be4Schools R\&D project implemented in the smart city of Águeda in Portugal. It comprised the former study in the country that examined the willingness of students (aged 15-21 years) to use e-bikes for daily trips to school and that gathered their preferences towards specific ICT related attributes.

Methods The methodology comprised a mobility survey and a stated-choice experiment (SC). The SC experiment gathered 2232 observations for modelling which were able to provide the relevant attribute informa'on trade-off between car travel, route and ebike features (with or without specific ICT equipment).

An extensive econometric analysis using was performed to assess the nature and extent of students' heterogeneity of preferences which also considered gender issues. The study aimed to contribute to the regional economic cluster on powered twowheels' industry \& innovation.

Results The absence of cycling infrastructures (segregated from main road) and the absence of cycle lanes in the road infrastructure were ranked as the first, second and third most important barriers, by $25.4 \%$ and $24.8 \%$ of the students, respectively.

The importance of a dedicated cycling route to school (segregated from main traffic) revealed to be critical as the odds of choosing an e-bike was found to be 6.5 times higher in comparison with the "no cycling infrastructure" option, ceteris paribus. This finding is aligned with the fact that cyclists would need to be exposed to high levels of motorized traffic in main roads and to increased perceived risks.

The market potential of e-bikes is likely to be higher if ICT features can be added to e-bikes as the odds of choosing an e-bike when it comes with the preferred ICT devices is 1.7 times higher than the opposed situation (e-bike without additional ICT devices). Conclusions Research results are interesting for mobility policies and industry as the possible integration of ICT equipment in ebikes may speed up the market uptake of this technology in smart cities. On the other hand, cycling infrastructures seem to be critical elements for increasing the demand for both conventional and e-bikes in the smart city of Águeda.
\end{abstract}

Keywords Electric bicycles $\cdot$ Sustainable mobility $\cdot$ Smart Cities $\cdot$ Stated preference $\cdot$ Behavioural modelling

This article is part of Topical Collection on Smart Cities and Transport Infrastructures

Elisabete Arsenio

elisabete.arsenio@lnec.pt

1 Department of Transport, LNEC, Lisbon, Portugal

2 Millenium BCP, Lisbon, Portugal

3 Faculdade de Ciências Médicas, Universidade Nova de Lisboa, Lisbon, Portugal

4 Faculdade de Ciências, Departamento de Estatística e Investigação Operacional, Universidade de Lisboa, Lisbon, Portugal

\section{Introduction}

The European Commission Transport White Paper [1] envisages that by 2050 only electric vehicles would circulate in cities, being the use of conventionally-fuelled vehicles halved by 2030 . Greenhouse gas emissions (GHG) from transport are expected to be reduced by $20 \%$ and $70 \%$, respectively until 2030 and 2050 (with respect to 2008 levels). The mentioned ambition and goals will imply technological changes and updates in city infrastructures. Behrendt [2] makes a strong case for the "smart velomobility" concept where cycling has an important role to play as a sustainable, active and networked mode in the context 
of Smart Cities and the Internet of Things. Electric bikes (ebikes) may contribute to transport decarbonisation but its full market potential remains to be clarified. Further research is needed to understand individuals' preferences and the nature of factors influencing effective changes from car use in cities to low carbon vehicles such as e-bikes.

Previous research [3] found that strategies for promoting ebike should differentiate between trip purpose and target groups (age segments). Overall, e-bikes are considered as a promising technology to reduce the external costs of transport, including traffic noise and air pollution effects [4]. As noted by Jones et al. [5], e-bikes could also help to increase accessibility for people who are unable or not willing to use conventional bicycles (e.g. elderly cyclists and people with physical limitations). In other cities, youngsters can represent a market segment for the early adopters of this technology and where behavioural changes can easily be promoted.

The research reported in this paper is built on the Be4Schools R\&D project implemented in the Portuguese city of Águeda which received in 2015 the smart city award. It benefited from the EU funded project SOLUTIONS - Sharing Opportunities for Low Carbon Urban Transportation that focuses on innovative sustainable urban mobility solutions in Europe and other regions in the world, namely in Asia, Latin America and the Mediterranean. The study reported in this paper examined the willingness of secondary school students to use e-bikes (age group 15-21 years) and their perceived cycling barriers, including the impact of non-existing cycling infrastructures in daily trips from home to/from school. The study included the participation of the city of Águeda, public schools in the municipally, secondary school students and their respective parents. The methodology comprised a mobility survey and a stated-choice experiment. The first part of the survey was designated as "Simplifying Cycling Mobility" and it aimed to understand students' travel patterns to school regarding mode choice, previous cycling experience, perceptions of barriers for not cycling and students' preferences for the future inclusion of ICT related attributes in e-bikes. Part two of the survey was designated as "Assessing students and their parents' preferences" which aimed to understand the preferred business models for the e-bike use, bicycle design attributes, including the most valued ones to be included in e-bikes and the preferred ICT equipments to be installed, thus accounting for household budget constraints. Part three comprised a stated-choice experiment that gathered 2232 observations around the relevant attributes' trade-off information between car travel and e-bike (with or without specific ICT equipment). An extensive econometric analysis was performed to assess the nature and extent of students' heterogeneity of preferences, also considering gender issues.

The reminder of this paper is organized as follows. Section 2 reviews the main determinants for cycling with conventional and electric bicycles within the context of home to/from school trips. Section 3 describes in detail the study including its design, exploratory data analysis and a multivariable logistic regression model. The significance level $\alpha=0.05$ was considered throughout the analysis. Statistical analyses were performed using the software IBM SPSS Statistics 21 and the R: A language and environment for statistical computing [6], for the multivariable logistic regression model. Finally, section 4 concludes and outlines the main implications for policy and practice.

\section{Determinants for influencing choice of conventional and electric bicycles for school trips}

Gössling and Choi [7] found that societal costs of driving a car are around six times higher than those of riding a bicycle. Overall, active travel modes such as cycling can be associated to significant health benefits if bicycle use is part of daily routines [8]. Although the majority of children and youth worldwide do not meet current physical activities (PA) guidelines of $60 \mathrm{~min}$ of daily moderate-to-vigorous PA, cycling to school is still uncommon in North America [9] as well as in other European countries such as Portugal. As outlined by Larouche et al., an unfavourable social norm for cycling and the poor maintenance of (low quality) cycling infrastructure may also explain the scarcity of cycling in the fall and winter in the majority of North American cities. Campbel et al. [10] noted that Canada have higher cycling rates than the United States due to the existence of better infrastructure which is confirmed in other research by Pucher and Buehler [11]. Regarding cycling using e-bikes, Fyhri and Fearnley [12] noted that more research is required to assess the potential of this vehicle as a means to reduce motorized travel.

Sustainable urban mobility policies shall promote bicycle use and modal shifts to decrease car traffic in smart cities. As earlier noted by Jones et al. [5], e-bikes could replace short and medium distance car journeys and help to promote more sustainable mobility patterns. This societal change requires knowledge on the factors that influence cycling behaviour and cross-modal shifts to allow the uptake of low carbon transport modes, including electric two-wheelers for utilitarian and commuting purposes. However, research on e-bike demand studies is still limited when compared to the one related to cycling using conventional bicycles. We found a small number of studies with indicators on possible determinants for the use of conventional bicycles and/ or can act as behavioural factors to promote shifts to e-bike in market context of school travel.

Heinen et al. [13] provide an extensive literature review of studies on the determinants for commuting by bicycle for utilitarian purposes. Their review also showed evidence that individuals decide sometimes whether or not to commute by bicycle by comparing cycling with other transport modes available tradingoff attributes such as costs, travel time and safety. Findings seem to point out the importance of a more comprehensive (multimodal) approach to cycling policies, able to address 
psychological factors (e.g. acting on individual's attitudes) and social opinions. The study developed by Emond and Handy [14] found that characteristics of the individual (e.g. gender, confidence), its social-environment (e.g. parents encouragement) and physical environment play a role when choosing to cycle to high school in Davis, California. This seems to point out that the mere existence of good cycling infrastructure is not sufficient to engage individuals in cycling. Overall, the social and behavioural impacts of emerging technological changes will also play a key role in planning mobility in smart cities.

Stewart et al. [15] reviewed qualitative and cross-sectional studies on active travel to school and identified eight common factors that influence cycling (and walking) to school in North America: distance to school, parental fear of traffic and crime, family schedule constraints and values (e.g. attitudes toward general physical activity), neighbourhood and family resources and culture, weather, and school characteristics. The review showed that features of the built environment such as lack of bicycle supporting facilities (including bicycle paths/infrastructures) and insufficient street network connectivity (along with other barriers in the route) were associated in some cases with lower cycling to school. Regarding distance to school among college students, Wuerzer and Mason [16] found that several socioeconomic characteristics (e.g. gender, age) and additional factors (e.g. car ownership) can mediate the impact of distance that one is likely to cycle. Nevertheless, the same authors also found a span of distance (between 3 to 4 miles) that one is willing to cycle that is not associated to car ownership.

In China several key forces were responsible for a significant market growth of electric two-wheelers vehicles (e-bikes and low-speed scooters) such as the rapid urbanization and air quality and traffic related problems. These externalities motivated a strong regulatory support for these vehicles, along with the deterioration of the bus transportation services and improvements in battery technology [17, 18]. In Europe, e-bikes are increasingly seen as a promising vehicle to replace car trips, especially in hilly cities such as the city of Águeda in Portugal and in those suffering from traffic congestion, excessive noise and air pollution. When compared to conventional bicycles these can enable a longer distance with less effort and reduced travel time. Lifecycle analysis demonstrates that riding e-bikes is also more environmentally friendly that driving cars $[19,20]$. On the other hand, electric vehicles are overall seen as a promising technology to decrease several road transport externalities [4], including the external costs of congestion, air pollution, traffic noise and climate change related effects assuming electric two-wheelers can penetrate the market at a large scale [21].

Popovich et al. [20] explored the experiences of 27 early adopters of e-bikes in the greater Sacramento, California. In their study, participants valued mostly the functional attributes of ebikes (speed, acceleration and less physical effort needs) in comparison to conventional bikes and these were used in commuting trips up to 20 miles each way. Security (risk of theft), safety aspects (due to higher speed and interaction with road traffic), bike weight and range anxiety were mentioned as key barriers. Regarding e-bike safety features, existing naturalistic studies on cycling provide mixed evidence and sometimes give contradictory findings on the real effects [22]. As such, one cannot conclude that e-bike users are more likely to be involved in crashes than conventional bike users. In the USA, Langford et al. [23] used a naturalistic study to compare safety behaviour of e-bike users and conventional bicycle riders and found no differences (with the exception of travel speed) between them. One online survey in North America by MacArthur et al. [24] found that ebike users owing a bike feel safer than if they were riding a conventional bike. As noted by Haustein and Moller [22], several factors can explain the risk differences of riding an e-bike in each country context such as the presence of cycling infrastructures, cycling norms and behaviour and existing regulations.

Astegiano et al. [25] found that in the city of Ghent in Belgium, e-bikes are highly used for commuting trips and the car is preferred for occasional trips that occur at most once per week. Moreover, the same study found that the problems related to conventional bicycles and e-bikes were similar and related to pavement conditions and road markings. Fyhri and Fearnley [12] study used a sample of 66 randomly selected participants (test users) and found that e-bikers increased the amount of their cycling (number of trips and distance travelled), in comparison to the control group, for both commuting and leisure trips. E-bike impacts in terms of number of trips (increase) were higher for females in comparison to male cyclists.

Campbel et al. [10], using a stated preference survey, examined the factors that influence the choice of conventional and electric bikes (public shared schemes) by adult consumers as a means to replace car use in Beijing, China. It shall be noted that in this city most non-motorway roads have a cycling lane. The study found that bike sharing schemes would promote mostly a shift from walking, individual (private) use of bicycle and ebiking and buses instead of replacing car trips. E-bike sharing were found to be more attractive to workers commuting outside of their neighbourhood and an option in over-subscribed bus routes with lower levels of service. Regarding the factors that influence choice, Campbel et al. [10] found that bicycle share and e-bike share are driven by distinct sets of factors: the former is more sensitive to effort and comfort related variables whereas the latter is more sensitive to contextual user heterogeneities (e.g. age, gender, education, income).

\section{Case study}

\subsection{Study design and sample}

The research included the design of an integrated survey that included the following main modules: 
- Simplifying mobility for using two wheelers, where the main objective was to gather information of the experienced/perceived barriers for cycling using either conventional bicycles and e-bikes;

- Assessing students' preferences, where the main purpose was to collect opinions towards bike use, most preferred business models and e-bike attributes;

- Stated-choice experiment, where students were asked to trade-off options in terms of bicycle/route attributes (e.g. with ICT features or not, percentage of route coverage with adequate cycling infrastructures) in comparison to the same trip to or from school using car (status quo).

Overall, the study aimed to explore the use of e-bikes by school students to replace car in trips to school. The city of Águeda along with the two secondary schools designated as "Adolfo Portela" and "Marques Castilho" were engaged in the project. Considering the last national population census [26] the total population of the municipality of Águeda is 47,729 inhabitants (49\% males and $52 \%$ females). This represents around $13 \%$ of the population of the region of Aveiro located in the Centre of Portugal. The municipality of Águeda has a population density of 142.4 inhabitants/ $\mathrm{km}^{2}$. For the same year, the total population of students (preschool, basic level 1, 2 and 3 and secondary schools) was 8892 students. The population of students in secondary schools (2014 data, PORDATA) is 1634 students. Considering the last mobility survey in the region of Aveiro conducted in 2011/2012 the average travel distance from home to school was $5.3 \mathrm{~km}$ or 3.3 miles (and the corresponding average distance for the return journey from school to home was $6.8 \mathrm{~km}$ or 4.2 miles).

In our research, the sample size comprised 248 students from two secondary schools in the city of Águeda. $43.5 \%$ were males and $51.2 \%$ were females whereas $5.3 \%$ did not answer this question. The stated-choice experiment collected 2232 observations about mode choice preferences (car or e-bike). The above sample was statistically representative of the student population at a $95 \%$ confidence level (margin of error of $2 \%$ ).

The students belonged to the secondary course levels 10th and 11th and were in the age segment 15-21 years (distributed as: $17.3 \% 15$ years old; $36.3 \%$ were 16 years old; $24.6 \%$, 17 years old; $8.9 \% 18$ years old; $4.4 \%$ were 19 years old; $1,6 \% 20$ or 21 years old and $6.9 \%$ did not answer). Regarding household composition, $44 \%$ of these students integrate households with four members and $19 \%$ of more than 5 members. $6.0 \%$ said to live alone or with one member of the family and $24.2 \%$ live with both parents. In the last National Population Census in 2011 [26], the average household size for the municipality of Águeda was 2.7, slightly higher than the national average which is 2.6.

\subsection{Perceived barriers for not cycling to school}

Considering students' travel patterns to school, $46.8 \%$ use car (as a passenger) in their daily travel to school, 27.8\% use the school bus, $11.7 \%$ walk, $9.7 \%$ uses train but no one uses bicycle to school (Fig. 1). $96.8 \%$ of students said to have learned how to ride a conventional bicycle and $15.3 \%$ said to use it regularly in the context of leisure trips. $94.4 \%$ use only one transport mode when travelling to school and $5.6 \%$ use more than one transport mode.

Students were asked to rank the most important barriers for not cycling to school. Figure 2 represents the top three barriers, i.e. the most important, second most important and third most important reasons for not cycling using conventional bicycles according to students' perceptions (the percentage of students is indicated for each case).

Figure 2 shows that the risk of accidents with other vehicles and the presence of hilly streets were perceived as the most important barriers for not cycling to school by $29 \%$ and $25.8 \%$ of the students, respectively. The absence of cycling infrastructures (segregated from main road) was ranked as the most important factor by $13.3 \%$ of the students. Also, the absence of cycle lanes (in a common road infrastructure) was ranked by $12.9 \%$ as the most important perceived barrier.

If the main perceived barriers are solved, $73.3 \%$ of the students said they would be willing to cycle to school. $50 \%$ of students would still prefer to use conventional bicycles and $23.3 \%$ would choose e-bikes (17\% of these prefer to rent one and the rest would purchase an e-bike).

Interest was focused on whether the sixteen barriers were perceived differently by the students of the two sampled schools. Non parametric Mann-Whitney tests were performed and their results concluded that the perceived importance levels in "Adolfo Portela" school (median =2) did differ significantly from the "Marques Castilho" school (median =1) as far as the barrier "Hilly streets" was considered, $U=3584.5, \mathrm{z}=-2.766$, $p=0.006$. The boxplots can be observed in Fig. 3 . This finding is consistent with the fact that access to school "Marques Castilho" involves routes of higher physical effort. The perceived importance levels of all the remaining barriers were not found to differ significantly between the two schools.

Students were questioned if they had already used the public electric bicycle of the city of Águeda which is designated as "beAgueda". When questioned about e-bike cycling, only $6 \%$ of the sample (15 students) said to have already used the electric

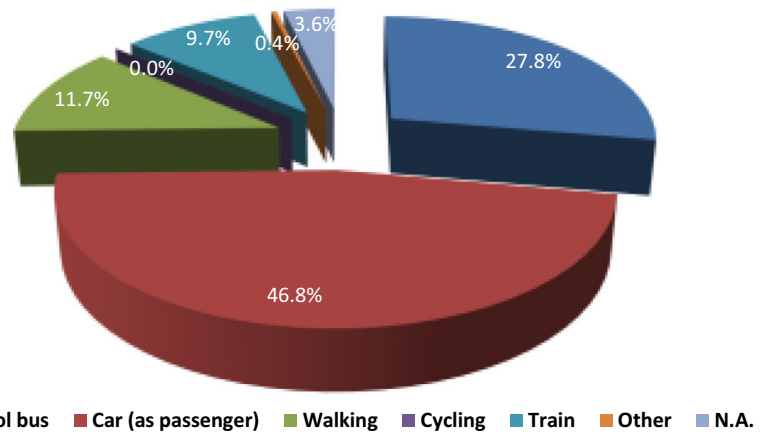

Fig. 1 Transport mode in home-school travel 


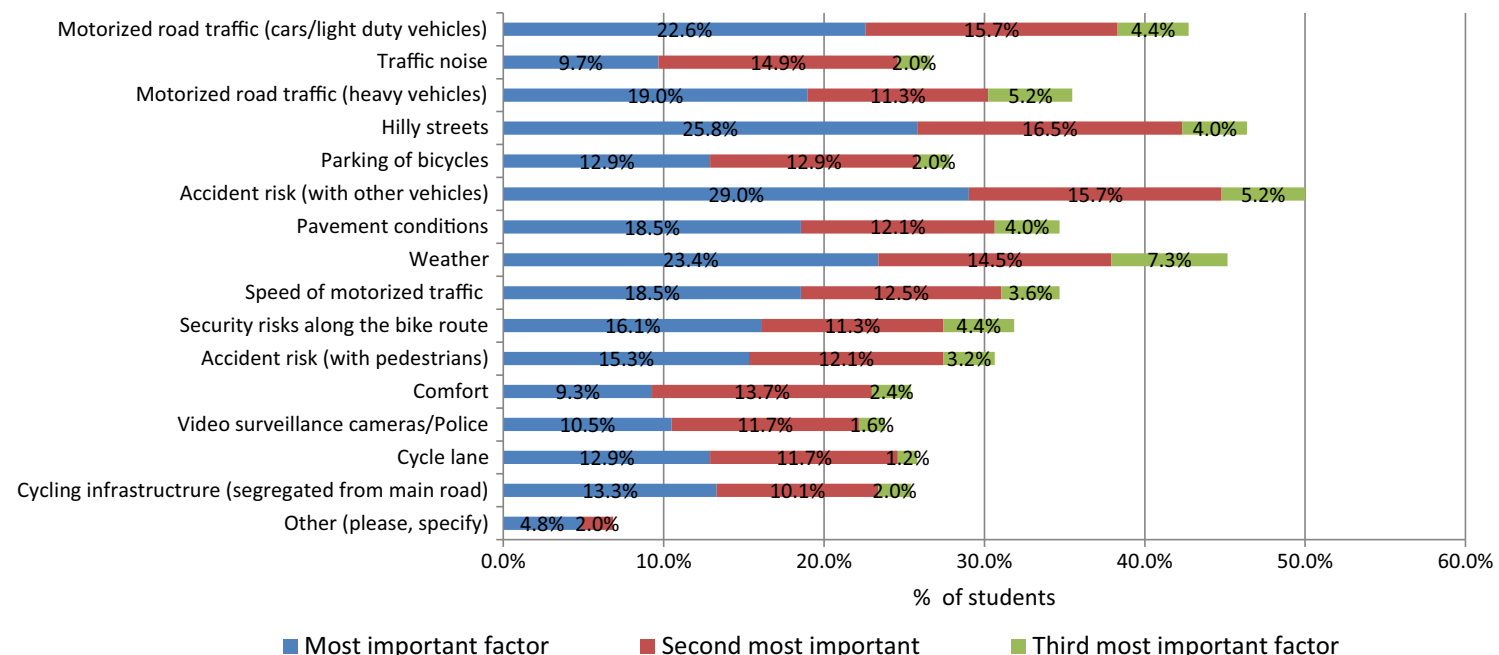

Fig. 2 Top-3 most important barriers (as perceived by school students) for not cycling to school

bicycle. We asked these 15 respondents about the most important experienced barriers when cycling with e-bikes and to rank these barriers by order of importance. Results of the top 3 barriers are presented in Table 1. Although this is a small sample of e-bikers, perceived barriers can act as complementary policy indicators. Table 1 shows that pavement conditions had a relative weight of $67 \%$ as these were considered as the most important barrier by six students and the second most important barrier by other four.

\subsection{E-bike attributes}

Students (and their respective parents) were asked to rank the attributes of the electric bikes they considered as first, second, third, etc. most important. Here we aimed to assess ICT related attributes such as bicycle connectivity between users (bicycle to bicycle connectivity or B2B) and additional equipment as valuable attributes to be included in e-bikes.

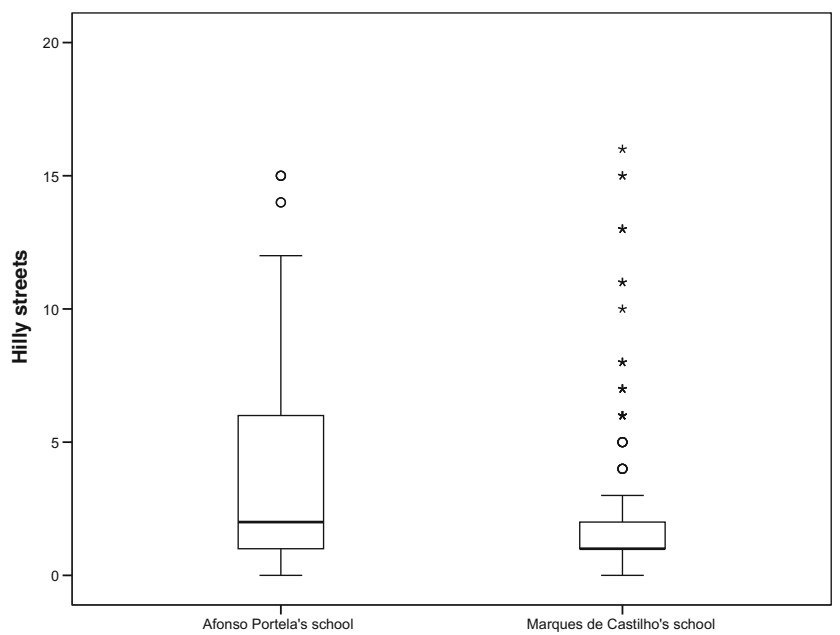

Fig. 3 Boxplots of the perceived importance levels of the "Hilly streets" barrier by the students of the Afonso Portela and Marques Castilho's schools
Figure 4 shows the top three most valued attributes (\% of students is indicated for each case). It shows that price of electric bicycles was considered as the most important attribute for $52.8 \%$ of the sample. Price was included in the top three most valued attributes with a weight of $65.3 \%$. The second most important attribute perceived for the e-bike was the reduced physical effort, ranked as most important by $23 \%$. Reduced physical effort was included in the top three most valued attributes with a weight of $42.7 \%$.

Regarding ICT related attributes and additional equipment, bicycle sensors to prevent road accidents with other vehicles and devices for personal security were considered as the most important attributes by respectively $23 \%$ and $21.4 \%$ of the respondents (if we consider the top three attributes these two features have a weight of $40.7 \%$ and $38.8 \%$ respectively). Bicycle connectivity with other bike users (B2B) was considered the most important attribute by $13.3 \%$ and it had a weight of $26.6 \%$ when considering the top three attribute features. Features related to devices to increase night time visibility and body protection devices were ranked as the most important e-bike attributes by $20.6 \%$ and $17.3 \%$. Regarding adding new equipment and ICT connection to conventional bicycles and e-bikes, $51 \%$ of the students said they would like to see these new features added.

When asked about their preferred bike business model (conventional versus e-bike), 50\% of the respondents said they would prefer to use a conventional bicycle. $31 \%$ of the respondents would prefer to ride an e-bike whereas $17 \%$ will prefer the renting model and the other $14 \%$ would prefer to purchase one e-bike. It is interesting to note that $19 \%$ of the respondents said they wouldn't choose neither the conventional nor the e-bike and would stay with their current mode choice. Students were also asked about their preferred bicycle model. The mountain model was the most voted $(52 \%)$ whereas $26 \%$ prefers the utilitarian model (bicycle with basket) and $21 \%$ said to prefer a personalized model design. The remaining students $(1 \%)$ would prefer a folded e-bike. Students' 
Table 1 Perceived barriers of students with e-bike experience

\begin{tabular}{lllll}
\hline Level of importance $\rightarrow$ Barrier & $\begin{array}{l}\text { Most } \\
\text { important }\end{array}$ & $\begin{array}{l}\text { Second most } \\
\text { important }\end{array}$ & $\begin{array}{l}\text { Third most } \\
\text { important }\end{array}$ & $\begin{array}{l}\% \text { of } \\
\text { students }\end{array}$ \\
\hline Weather & 8 & 1 & 1 & $67 \%$ \\
Pavement conditions & 6 & 4 & 0 & $67 \%$ \\
Motorized road traffic (light duty) & 5 & 2 & 2 & $60 \%$ \\
Traffic noise & 5 & 2 & 0 & $60 \%$ \\
Accident risk (with other vehicles in the road) & 5 & 3 & 0 & $53 \%$ \\
Traffic speed & 5 & 3 & 0 & $53 \%$ \\
Accident risk (with pedestrians) & 5 & 2 & 0 & $47 \%$ \\
\hline
\end{tabular}

preferences for the mountain bicycle model are somehow expected due to the existing mountain biking market in the region for sport purposes. Also, considering the European bicycle market, mountain bikes are the most important range with a market share of $28.5 \%$ [27].

Before the SP experiment, the respondents that had confirmed to be willing to cycle if ICT devices are added to the bicycle, were asked to choose their preferred ICT devices (more than one option could be chosen). Amongst the additional equipment available for the e-bikes, the most voted one consisted of an ICT connection between different bicycles (which included an integrated GPS system enabling the calculation of the shortest route), having obtained $64 \%$ of the votes. The second most popular choice of equipment was a device that counts the calories spent during the ride (53\%). It was then followed by the option of considering several equipments together, that is, an option that includes an ICT system, a calories counter, a Bluetooth device and a cooperative bicycle system ( $50 \%$ of the votes). We have tested if there were differences in the preferences between students of the two schools as the school Marques Castilho is considered as more related to technological courses. None of the several mentioned options of equipment seemed to have differed between the students of the two schools (non-parametric MannWhitney tests, $p=0.074$ for ICT device, $p=0.125$ for the calories measurement, $p=0.697$ for the Bluetooth device, $p=0.463$ for the bicycle cooperative and $p=0.345$ for the total of the four devices combined).

\subsection{Stated-choice experiment}

The last part of the mobility survey included a stated-choice (SC) experiment where future alternative scenarios for using electric bicycles in students' trips to school were explored. The number and type of attributes in the SP design took into account the respondent's tendency to simplify tasks that they are not familiar with or are excessive. Also, presenting too many attributes may cause difficulties on making trade-offs [28]. The present study used a fractional factorial design and an orthogonal main effect's plan [29]. This means that the selected attributes are not correlated (each attribute is orthogonal to the others), although in real life they can be. Nine random scenario options (i.e. possible scenarios for riding an e-bike) were presented and described as combinations of four main variables (and attribute levels) as follows:

a) Cost of e-bike levels: same rental cost as the school travel monthly pass (reference cost level), $25 \%$ higher, $50 \%$ higher;

b) Route coverage by cycling infrastructures: None (reference level or status quo); $50 \%$ of the route home to/from

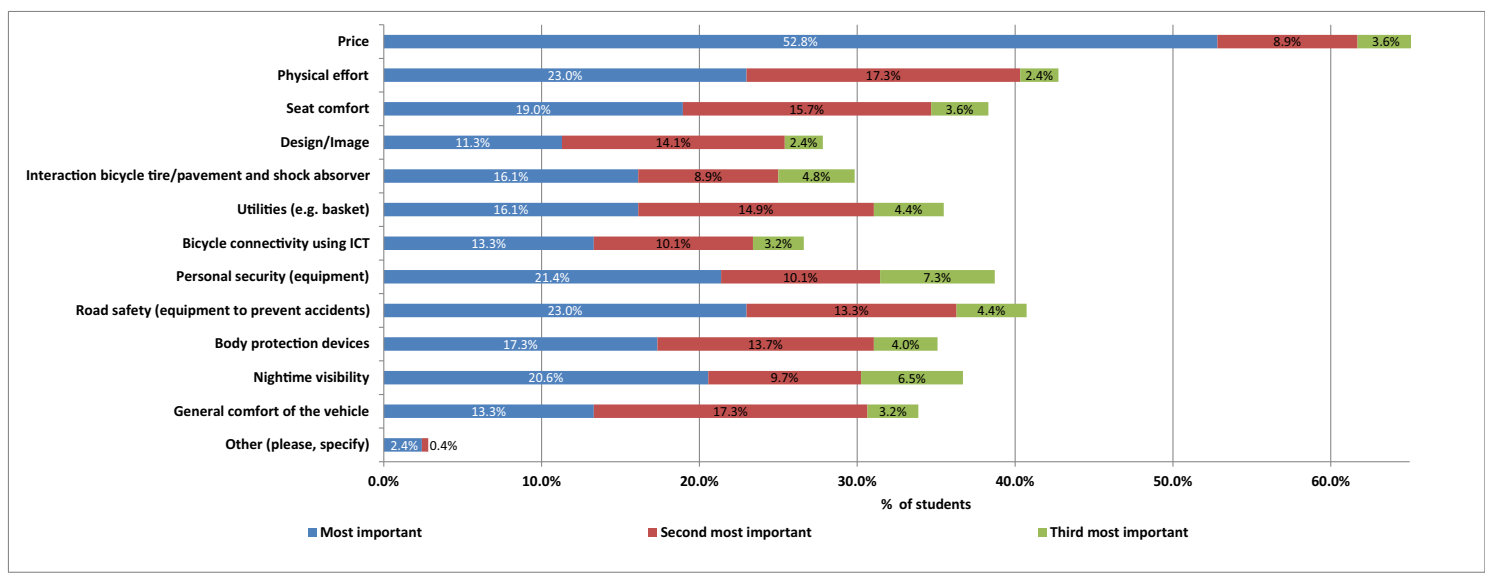

Fig. 4 Most important attributes of electric bicycles 
school is covered; $100 \%$ of the route home to/from school is covered;

c) Presence of ICT equipment for e-bike connectivity, GPS, etc. (most preferred devices): Yes; No (reference category);

d) Travel time: increase of $10 \mathrm{~min}$ from the current situation (reference level); increase of $20 \mathrm{~min}$; increase of $30 \mathrm{~min}$.

During the experiment, respondents were asked if they would choose an e-bike to travel in each situation (scenario) or prefer the car choice option. During the modelling work several covariates were explored to estimate logistic regression models. As such, the model included variables such as parents' socio-economic group (high; medium (reference category) and low) and school type (Adolfo Portela School (reference category) and Marques Castilho School/ technological related school). The students' gender was introduced in the model in order to gain some understanding of how it might influence the outcome variable. It was found that there were no statistical significant gender differences when the choice between an electric bicycle or the car of her/his parent was concerned $(\mathrm{OR}=0.994$, with $95 \%$ CI $(0.706,1.403), p=$ 0.973). Therefore, it was decided not to account for the gender on the subsequent models considered.

The parameter estimates of the model that provided the best fit to the data are represented in Table 2, these include the model's estimated coefficients, odds ratios (OR) and corresponding 95\% confidence intervals (CI) and $p$-values. Table 2 shows that if the e-bike operational cost is $25 \%$ or $50 \%$ higher than the school transport monthly pass, the odds of choosing an e-bike will decrease by $85 \%$ and $94 \%$, respectively, when compared with an e-bike cost equal to the school transport pass. Also, an increasing cycling time to school has a negative impact with the odds of choosing an e-bike decreasing by $69 \%$ and $92 \%$ when the travelling time is $20 \mathrm{~min}$ or
30 min higher, respectively, when compared with a 10 min' increase in travel time. Other important findings from the model presented in Table 2 can be summarized as follows:

- The odds of choosing an e-bike are two times higher for students who have had at least 10 years of conventional bicycle experience (for leisure purposes) compared with students who have had less than 10 years as previous experience.

- Regarding the ICT features, the odds of choosing an ebike are 1.7 times higher if it comes with an ICT component as opposed to having no such component, however, this statement is not statistically significant $(p=0.097)$. This finding is particularly important for the bicycle industry in the region of Águeda.

- The odds of choosing an e-bike were 2.3 and 6.5 times higher if the length of the route has an overall coverage of $50 \%$ or $100 \%$, respectively, as compared with no coverage for its total length.

- The odds of choosing an e-bike were 2.7 times higher for students whose parents belonged to a high socioeconomic group compared with students whose parents belonged to a medium socio-economic group. Nevertheless, these odds decrease by $20 \%$ when the students belong to a low socio-economic family compared with a medium socio-economic one.

Each of the above statements is valid when the adjustments for all of the remaining variables in the model were considered.

The overall rate of correct classification, employing the usual 0.5 cutpoint, is estimated as $76.3 \%$, with $91.7 \%$ correct classification of car choice (specificity), but only $28.5 \%$ correct classification in the group that actually choose the e-bike (sensitivity). According to Hosmer et al. [30], classification is sensitive to the relative sizes of the two component groups and
Table 2 Covariates, coefficient estimates, OR, 95\% CI and $p$ values of the multiple logistic regression model

\begin{tabular}{lcccc}
\hline Covariate & Coefficient estimate & OR & 95\% CI & $p$-value \\
\hline Intercept & 0.832 & 2.299 & $(1.105,5.343)$ & 0.0350 \\
Cost ( $>25 \%)$ & -1.916 & 0.147 & $(0.076,0.245)$ & $<0.001$ \\
Cost ( $>50 \%)$ & -2.775 & 0.062 & $(0.024,0.127)$ & $<0.001$ \\
Length of cycling infrastructure (50\%) & 0.824 & 2.281 & $(1.250,4.686)$ & 0.0116 \\
Length of cycling infrastructure (100\%) & 1.866 & 6.465 & $(3.493,13.924)$ & $<0.001$ \\
ICT equipment (yes) & 0.548 & 1.730 & $(0.847,3.307)$ & 0.0968 \\
Cycling travel time (20 min higher) & -1.156 & 0.315 & $(0.178,0.511)$ & $<0.001$ \\
Cycling travel time (30 min higher) & -2.483 & 0.084 & $(0.038,0.158)$ & $<0.001$ \\
School (Marques Castilho) & 0.401 & 1.493 & $(1.032,2.179)$ & 0.0355 \\
Previous e-bike experience (>10 years) & 0.712 & 2.037 & $(1.436,2.915)$ & $<0.001$ \\
Socio-economic group (high) & 0.980 & 2.664 & $(1.690,4.220)$ & $<0.001$ \\
Socio-economic group (low) & -0.226 & 0.798 & $(0.546,1.158)$ & 0.2381 \\
\hline
\end{tabular}

Log Likelihood: $\mathbf{- 4 3 5 . 8 9 2}$

McFadden $\mathrm{R}^{2}$ : 0.222 
always favours classification into the larger group (i.e. the students who chose the car), a fact that is also independent of the fit of the model. The same authors claim that a better and more complete description of the classification accuracy is the area under the ROC curve. This curve is obtained by plotting the probability of detecting a true e-bike choice (sensitivity) and a car choice (1-specificity) for an entire range of possible cutpoints. Figure 5 shows the ROC curve obtained by the model described in Table 2 .

The area under the ROC curve provides a measure of the model's ability to discriminate between those students who choose the e-bike option versus those who choose using their parents' car. As Fig. 5 shows, the area under the ROC curve is 0.802 which according to Hosmer et al. [30] indicates an "excellent discrimination". Figure 5 also shows the optimal cutpoint for classification (0.163) and its corresponding sensitivity, specificity, positive and negative predictive values.

\section{Conclusions}

The research reported in this paper represents the former study in the country that examined the willingness of secondary school students to use e-bikes in their daily travel to school and, additionally, that assessed the role of additional ICT related devices (B2B connectivity, sensors to detect presence of vehicles in the proximity, etc.) to increase the likelihood of choosing the e-bike and contribute to low carbon transport. In the context of the city of Águeda, $46.8 \%$ of students travel to school as a car passenger and although $27.8 \%$ uses the school bus no one uses conventional bicycles in commuting to school. This mobility pattern where cycling to school is almost inexistent also reflects the situation at the country level.

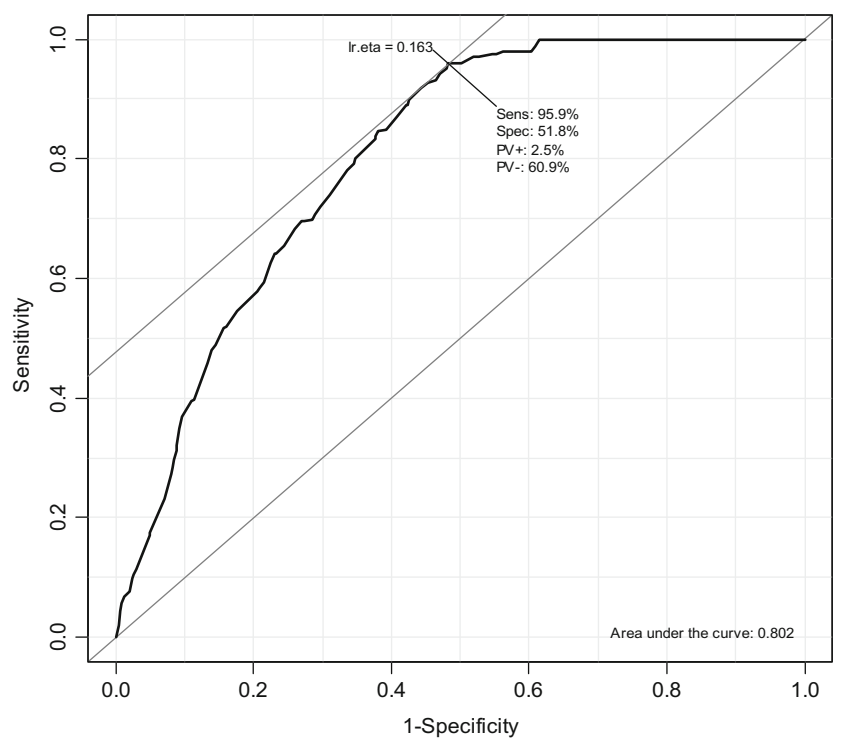

Fig. 5 ROC curve for the multiple logistic regression model in Table 2
The study contributed to understand which barriers are perceived by students as the most important ones for them not to cycle to school and to assess the specific role of cycling infrastructures. It was found that the risk of accidents with other vehicles and the presence of hilly streets were the most important perceived barriers for not cycling to school. The absence of cycling infrastructures (segregated from main road) and the absence of cycle lanes in the road infrastructure were ranked as the first, second and third most important barriers, by $25.4 \%$ and $24.8 \%$ of the students, respectively.

The stated-choice experiment considered 2232 observations for estimating multiple logistic regression models. The importance of a dedicated cycling route to school (segregated from main traffic) revealed to be critical as the odds of choosing an e-bike was found to be 6.5 times higher in comparison with the "no cycling infrastructure" option, ceteris paribus. This finding is aligned with the fact that cyclists would need to be exposed to high levels of motorized traffic in main roads and to increased perceived risks. Therefore, implementing cycling infrastructures to school will definitely facilitate the use of e-bikes in this context. Also, the market potential of e-bikes is likely to be higher if ICT features can be added to e-bikes as the odds of choosing an e-bike when it comes with the preferred ICT devices is 1.7 times higher than the opposed situation (e-bike without additional ICT devices). This finding is important for the industry in the region wanting to increase the uptake of these new technologies and e-bikes as a means to contribute to transport decarbonisation goals.

Overall, a significant market innovation potential for cycling remains to be explored in the context of school travel in the country that requires solving a set of barriers of varying complexity to address user needs.

Acknowledgments The authors express their thanks to the City of Águeda for all the support during the fieldwork.

Publisher's Note Springer Nature remains neutral with regard to jurisdictional claims in published maps and institutional affiliations.

Open Access This article is distributed under the terms of the Creative Commons Attribution 4.0 International License (http:// creativecommons.org/licenses/by/4.0/), which permits unrestricted use, distribution, and reproduction in any medium, provided you give appropriate credit to the original author(s) and the source, provide a link to the Creative Commons license, and indicate if changes were made.

\section{References}

1. European Commission - EC (2011) Roadmap to a single European transport area - towards a competitive and resource efficient transport system. European Commission, Brussels

2. Behrendt F (2016) Why cycling matters for smart cities. Internet of bicycles for intelligent transport. J Transp Geogr 56:157-164

3. Wolf A, Seebauer S (2014) Technology adoption of electric bicycles: a survey among early adopters. Transp Res A 69:196-211 
4. Jochem P, Doll C, Fichtner W (2016) External costs of electric vehicles. Transp Res D 42:60-76

5. Jones T, Harms L, Heinen E (2016) Motives, perceptions and experiences of electric bicycle owners and implications for health, wellbeing and mobility. J Transp Geogr 53:41-49

6. R Core Team (2016) R: a language and environment for statistical computing. R Foundation for Statistical Computing, Vienna http:// www.R-project.org/

7. Gössling S, Choi AS (2015) Transport transitions in Copenhagen: comparing the costs of cars and bicycles. Ecol Econ 113:106-113

8. Arsenio E, Ribeiro P (2015) The economic assessment of health benefits of active transport. In: Attard M, Shiftan Y (eds) Sustainable urban transport (transport and sustainability, volume 7). Emerald Group Publishing Limited, UK, pp 1-22. https://doi. org/10.1108/S2044-994120150000007011

9. Larouche R, Stone M, Buliung R, Faulkner G (2016) "I'd rather bike to school": profiling children who would prefer to cycle to school. J Trans Health 3:377-385

10. Campbel A, Cherry C, Ryerson M, Yang X (2016) Factors influencing the choice of shared bicycles and shared electric bikes in Beijing. Transp Res C 67:399-414

11. Pucher J, Buehler R (2006) Why Canadians cycle more than Americans: a comparative analysis of bicycling trends and policies. Transp Policy 13(3):265-279

12. Fyhri A, Fearnley N (2015) Effect of e-bikes on bicycle use and mode share. Transp Res D 36:45-52

13. Heinen E, van Wee B, Maat K (2010) Commuting by bicycle: an overview of the literature. Transp Rev 30(1):59-96

14. Emond CR, Handy SL (2012) Factors associated with bicycling to high school: insights from Davis, CA. J Transp Geogr 20:71-79

15. Stewart O, Moudon A, Claybrooke C (2012) Common ground: eight factors that influence walking and biking to school. Transp Policy 24:240-248

16. Wuerzer T, Mason S (2015) Cycling willingness: investigating distance as a dependent variable in cycling behaviour among college students. Appl Geogr 60:95-106

17. Weiner J, Ma C, Chery C (2006) The transition to electric bikes in China: history and key reasons for rapid growth. Transportation 34: 301-318
18. Weiner J, Ogden J, Sperling D, Burke A (2008) The future of electric two-wheelers and electric vehicles in China. Energ Policy 36:2544-2255

19. Cherry C, Weinert J, Xinmiao Y (2009) Comparative environmental impacts of e-bikes in China. Transp Res D 14:281-290

20. Popovich N, Gordon E, Shao Z, Xing Y, Wang Y, Handy S (2014) Experiences of electric bicycle users in the Sacramento, California area. Travel Behav Soc 1:37-44

21. Weiss M, Dekker P, Moro A, Scholz H, Patel M (2015) On the electrification of road transportation - a review of the environmental, economic, and social performance of electric two-wheelers. Transp Res D 41:348-366

22. Haustein S, Moller M (2016) E-bike safety: individual-level factors and incident charactristics. J Trans Health 3:386-394

23. Langford B, Chen J, Cherry C (2015) Risky riding: naturalistic methods comparing safety behaviour from conventional bicycle riders and electric bike riders. Accid Anal Prev 82:220-226

24. MacArthur J, Dill J, Person M (2014) Electric bikes in North America: results of an online survey. Transport research. Theatr Rec 2468:123-130

25. Astegiano $\mathrm{P}$, Tampère $\mathrm{C}$, Beckx $\mathrm{C}(2015) \mathrm{A}$ preliminary analysis over the factors related with the possession of an electric bike. Trans Res Procedia 10:393-402

26. INE (2011) XV Recenseamento Gerald a População CENSOS 2011. Lisboa, INE

27. CONEBI - Confederation of the European bicycle industry (2014). European bicycle market. Industry and market profile (2013 Statistics), CONEBI: Belgium

28. Bates J (1998) Reflections on stated preference: theory and practice. In: Ortuzar, J. D. Hensher and S. Jara-Diaz (eds). Travel behaviour research: updating the state of play, chapter 6 . Pergamon, UK, pp 89-103

29. Kocur G, Adler T, Hyman W, Aunnet B (1982) Guide to forecasting travel demand with direct utility assessment. Technical report, United States Department of Transportation, Washington DC, USA

30. Hosmer D, Lemeshow S, Sturdivant R (2013) Applied logistic regression, 3rd edn. Wiley Series in Probability and Statistics, Wiley, New Jersey 